\title{
Reviews, reviewers and reviewing
}

\author{
Ancuța Zazgyva $^{1} \cdot$ Elizaveta Kon $^{2} \cdot$ Cyril Mauffrey $^{3}$ - Andreas F. Mavrogenis ${ }^{4}$. \\ Marius M. Scarlat ${ }^{5}$
}

Published online: 2 December 2016

(C) SICOT aisbl 2016

The November Editorial of Clinical Orthopaedics and Related Research [1] authored by our distinguished colleague Seth S. Leopold from Seattle enlightened our vision about the peer review process and challenged our minds. Reviewers are key characters in the scientific production process; therefore we positioned ourselves as evaluators, as we are all reviewers at some point. Since we found this experiment valuable, we proposed to share it with you and ask rhetorically: Could you still recall the first time you've been invited by an editor to review a scientific paper? It is usually pretty exciting, but why should you spend time with it? Most of us are prone to accept an invitation to review, as it acknowledges our expertise in a specific domain. The call to serve as a referee for a manuscript is an honour, for sure, but also a responsibility; however, at the bottom line a reviewer improves gradually, raising his or her credentials and curricular scores, refining writing skills and keeping updated with new and exciting findings in science.

Any submitted article is usually first checked by a Publishing Assistant to verify whether the submission fits the Journal's standards, if the references are formatted according to the Instructions for Authors and if the word count is within the allowed limits. Then, the papers that qualify are sent to the Editor. The Editor evaluates the article to see if it

Marius M. Scarlat

mscarlat@gmail.com

1 University of Medicine and Pharmacy Târgu Mureș, Târgu Mureş, Romania

2 Rizzoli Orthopaedic Institute, Bologna, Italy

3 Denver Health Medical Centre, Denver, CO, USA

4 National and Kapodistrian University of Athens, School of Medicine, Athens, Greece

5 Clinique St Michel, Toulon, France falls within the Journal's scope and if it is prepared according to the Instructions for Authors. If the paper matches these criteria, it is sent to Reviewers for peer review [2]. If they agree to review, they are encouraged to perform a thoughtful review, which must be honest, free of bias, confident, and polite, and suggests a decision to the Editor. Reviewers are volunteers, generally chosen for their personal experience, and many are senior academicians and researchers $[2,3]$. Still, before hitting the "accept" button we may recall some points, as Kotsis and Chung revealed in their very useful quick guide on reviewing $[4,5]$. Namely, questioning if your individual experience is appropriate for serving as a referee for the paper proposed and you can provide a timely and unbiased opinion. If you feel qualified to evaluate the strengths and weaknesses of the manuscript and can do so in the required time, without conflicts of interest, you may accept the request and do your best to deliver a thoughtful and thorough review. But then, what next? Depending on how busy and/or organized you are, you might start thinking about that review assignment right away or perhaps a few days later. You should bear in mind that a proper, carefully prepared review might take up quite a lot of your time, so plan ahead to be able to dedicate a few hours for the work. The current 'push system' of review where the editor assigns a reviewer without having an insight into the reviewers time constraints may need to be modified to a 'pull system' where the reviewer shows his availability and willingness to review a manuscript at a specific time point. This revolution would require a drastic modification of the culture, computer systems and workflow currently in place. In addition, it is possible that the good reviewers may never be available to flag their desire to perform a review [6]. Now you face the next question: what makes a good review?

Although not perfect, peer review is still at the heart of scientific quality control, and is a way for each of us to 
contribute to our field. As with anything in life, you should consider things from an empathic point of view - if you were the author of that article, what would you expect from a review and a reviewer? As we all have been in the author position, this one is easy to answer: a review should be performed in the required time, objective, helpful, positive in providing the best ways to improve the paper - even rejected, and presented in a respectful manner for the author who spent so much time in providing the data. All those aspects are important, as a quick reply facilitates swift revisions/publication or, in case of a rejection, it wastes the least amount of time before the manuscript can be submitted to a different journal or to be resubmitted after substantial changes. A review must be objective; evaluation on subjective terms is debatable, personal feelings from known peers should be avoided - this is why major journals, including "International Orthopaedics" use a double blinded review system. The ideal peer review should also be helpful, offering constructive criticism, even if the final recommendation is for rejection. A detailed, thoughtful and well-constructed bullet pointed list of comments will be very appreciated by any author, as it makes it easier to answer all raised concerns.

In the review process, the fundamental questions to be answered in the reviewing process are: (1) Is the rationale/hypothesis, original, and interesting? (2) Does and how the study merits publication? (3) Are the methods valid? and (4) Do data and literature support the conclusions? Would they challenge and provoke readings, citations, impact? [2, 3, 7]. Providing respectful comments as a reviewer is paramount, regardless of the manuscript's overall quality; the role of the reviewers is to advise the Editor and eventually to help the authors to improve their paper. Most authors made a substantial effort to conduct their study and prepare their paper, and usually they present the best work for evaluation; keeping a collegial and respectful tone, showing appreciation of these efforts and the trust bestowed upon you is mandatory. A quick tip on language and grammar: most journals are opened for an international audience and authors, so as a reviewer you should only bring up language problems if they make it difficult to understand the paper, but do so politely; you can always signal these deficiencies in your confidential comments to the editor.

And another important mention: Yes! - reviewing is time consuming, Yes - the number of Journals sending you papers to review start to increase quickly and keep increasing all the time and you are constricted sometimes to feel badly to refuse something... But every time you make a review you learn something. You learn not only about new research before others (and this is just a great privilege), but also every review you perform improves your skills and refines your methodology and style. So, if you think about it deeply - it is not so altruistic a commitment after all...

How would you set out accomplishing such a seemingly daunting task? Ideally you should be coached into it by a senior scientist/mentor. There is also a lot of information that you could find on the subject, teaching you the methods you need to go about in doing a suitable review, as well as the proper conduct of a reviewer $[8,9]$. Recently, other helpful instruments have emerged that can assist you in your reviewing endeavours, such as the newly launched peerreviewer tool from our colleagues [1]. However, at the bottom of your mission is one decision: your recommendations to the editor - accept, revise or reject. An excellent reviewer should act as an 'advocate for the author', but keep in mind that the editor trusts you to provide a recommendation that can benefit the journal's readership, whilst being in line with the publication's aims and scope. Your decision must reach a delicate balance between these two important aspects. Reviewing manuscripts is a skill that can be practiced and improved. As Benos et al. explained, a good reviewer is characterised by fairness, thoroughness and integrity [9]. Although the majority of referees did not have a formal training in the 'art of reviewing' they still continue to selfishly contribute their time and effort in making sure that more quality research reaches its target audience. This work and commitment is wellappreciated by "International Orthopaedics" and all the scientific community [10]. Every year our valuable reviewers are acknowledged in a special list published as Open Access in the February issue and this is a modest tribute that we can offer to a beautiful and meaningful group of individuals that progress in scientific endeavours.

\section{References}

1. Leopold SS (2016) Editorial: CORR's new peer-reviewer tool-useful for more than peer reviews. Clin Orthop Relat Res 474(11): 2321-2322

2. Mauffrey C, Scarlat MM, Pećina M (2014) Setting standards for medical writing in orthopaedics. Int Orthop 38(1):1-5

3. Mavrogenis AF, Quaile A, Scarlat MM (2016) Attractive papers and accurate English. Int Orthop 40(4):649-651

4. Kotsis SV, Chung KC (2014) Manuscript rejection: how to submit a revision and tips on being a good peer reviewer. Plast Reconstr Surg 133(4):958-964

5. Hak DJ, Giannoudis P, Mauffrey C (2016) Increasing challenges for an effective peer-review process. Eur J Orthop Surg Traumatol 26(2):117-118

6. Lovejoy TI, Revenson TA, France CR (2011) Reviewing manuscripts for peer-review journals: a primer for novice and seasoned reviewers. Ann Behav Med 42(1):1-13

7. Scarlat MM, Mavrogenis AF, Pećina M, Niculescu M (2015) Impact and alternative metrics for medical publishing: our experience with International Orthopaedics. Int Orthop 39(8):1459-1464

8. Einarson TR, Koren G (2012) To accept or reject? A guide to peer reviewing of medical journal papers. J Popul Ther Clin Pharmacol 19(2):e328-333

9. Benos DJ, Kirk KL, Hall JE (2003) How to review a paper. Adv Physiol Educ 27(1-4):47-52

10. Scarlat MM, Hinsenkamp M, Quaile A, Pecina M (2016) International Orthopaedics is $4 \mathrm{O}$ years old! Int Orthop $40(8)$ : $1563-1569$ 